\title{
MCNPX Simulation for Radiation Dose Absorption of Anatomical Regions and Some Organs
}

\author{
E.E. Altunsoy ${ }^{a, b, c, *}$, H.O. Tekin ${ }^{c, d}$, A. Mesbahi ${ }^{e}$ And I. AKKurt ${ }^{b}$ \\ ${ }^{a}$ Üsküdar University, Vocational School of Health Services, Medical Imaging Department, Istanbul 34662, Turkey \\ ${ }^{b}$ Süleyman Demirel University, Science and Art Faculty, Physics Department, Isparta 32260, Turkey \\ ${ }^{c}$ Üsküdar University, Medical Radiation Research Center (USMERA), Istanbul 34662, Turkey \\ ${ }^{d}$ Üsküdar University, Vocational School of Health Services, Radiotherapy Department, Istanbul 34662, Turkey \\ ${ }^{e}$ Tabriz University of Medical Sciences, Medical School, Medical Physics Department, Tabriz, Iran
}

\begin{abstract}
Radiation physics has been an important subject for researchers as it is used especially in medical fields for different purposes and also due to its hazardous effects. This study is aimed to investigate the mass attenuation coefficient of some organs and the radiation absorption effects of adipose tissue on computed tomography scan in different radiological energies using MCNPX (version 2.6.0) general purpose Monte Carlo code. This simulation was done by selecting organs from the cranium, thorax and abdominal anatomical regions. Additionally, the mass attenuation coefficient of adipose tissue was calculated to determine the relationship between radiation reduction rates of anatomical regions and adipose tissue thickness. On the other hand, the radiation attenuation quantities of the selected computed tomography regions were calculated, showing the effect of adipose tissue on these measurements. The MCNPX simulations were compared with the results obtained by XCOM data to ensure validation of this simulation geometry and a good agreement was observed.
\end{abstract}

DOI: 10.12693/APhysPolA.137.561

PACS/topics: radiation dose, computed tomography, MCNPX, mass attenuation coefficient

\section{Introduction}

Computed tomography (CT) is a diagnostic device that visualizes the body in cross-sectional images using $\mathrm{X}$-rays. The principle of creating a CT image is that the amount of light that passes through the anatomical region of the patient, which is emitted from the X-ray source, is measured by the detectors on the device. Although the radiation dose used during computed tomography imaging is directly related to image quality, it can also cause biological effects of radiation in patients. Types of ionizing radiation (such as X-rays and gamma rays) cause detrimental effects in living cells, tissues, and organisms. Therefore, the protection against detrimental radiation is important. The interaction of the gamma ray with a medium is associated with the Lambert-Beer law $I=I_{0} \mathrm{e}^{-\mu t}$, where $I$ and $I_{0}$ represent the light intensity after it passes through the target material and the initial light intensity coming to the target material, respectively. Next, $\mu\left(\mathrm{cm}^{-1}\right)$ is linear attenuation coefficient of the material, and $t(\mathrm{~cm})$ represents the thickness of the target material. The contrast of the CT image depends upon photon attenuation of body regions [1]. The literature review showed that various studies have been performed regarding attenuation properties of different material environments. Akkurt et al. [2] have calculated the linear

\footnotetext{
* corresponding author; e-mail: elifebru.altunsoy@uskudar.edu.tr
}

attenuation coefficients and total mass attenuation coefficients of gamma rays for barite, marble, and limra using XCOM. They compared XCOM results with experimental results measured by $\mathrm{NaI}$ detector. Tekin et al. [3] have investigated the effect of the shielding properties of $\mathrm{WO}_{3}$ and $\mathrm{PbO}$ on the radiation mass attenuation coefficients using MCNPX (version 2.4.0). Tekin et al. [4] have conducted a study demonstrating the validation of MCNPX results with experimental results of mass attenuation coefficients of mixtures of cement, gypsum, and gypsium- $\mathrm{PbCO}_{3}$.

In recent years, the increasing popularity of simulation methods has involved the difficulty of working on living organs and the fact that these studies are not always possible. This has contributed to the research on living organs by the simulation method. DeMarco et al. [5] have developed a graphical user interface that automatically adjusts the MCNP4A geometry and radiation source requirements for a three-dimensional Monte Carlo simulation using CT data. This user interface allows homogeneous or heterogeneous dose calculations in individual voxel elements. Yoriyaz et al. [6] have developed the MCNP-4B code with the Monte Carlo method as a new three-dimensional dose calculation approach in the use of radioimmunotherapy internal radiation source. Stoeckelhuber et al. [7] have conducted a study on the measurement of the radiation dose at the operator's hand during interventions conducted under the guidance of CT fluoroscopy.

This study showed that the procedure should be completed as soon as possible during the $\mathrm{CT}$ fluoroscopic procedure, and established the importance of length 
of the needle holder and radiation shielding materials. Salvado et al. [8] have aimed to develop and validate a method for calculating radiation doses during CT examinations using a voxel-based Monte Carlo simulation method. Peixoto et al. [9] have studied the creation of a tomographic model of male wistar rats using CT images to use in radiation transmission studies. Morato et al. [10] have aimed to develop an automated methodology to calculate the dose received by a patient exposed to ionizing radiation during CT scans. Tekin et al. [11] have calculated the mass attenuation coefficients of the human body organs using the MCNPX Monte Carlo code and compared the results with XCOM, FLUKA, GEANT4, and NIST data. However, no study has been performed on attenuation properties anatomical regions at CT scan energies. There are also a number of studies done for medical applications [12-16].

This has encouraged us to generate a simulation setup and investigate the attenuation properties considering the chemical properties of investigated organs and tissues.

\section{Materials and method}

In this study, the mass attenuation coefficients of some organs in anatomical regions of CT scans and the amount of radiation attenuation due to the thickness of these regions were investigated and the results were compared with standard XCOM data. Monte Carlo N-particle Transport Code Extended (MCNPX version 2.6.0) was utilized for calculating the mass attenuation coefficients and compared with XCOM-NIST (National Institute of Standards and Technology) data.

\subsection{MCNPX Monte Carlo code}

In this study, cranium, thorax, and abdominal regions were defined in the input file as the anatomical region. On the other hand, brain tissue, lung tissue, and liver tissue were selected from these anatomic regions, respectively, in order to calculate radiation mass attenuation coefficients using MCNPX (version 2.4.0). In addition, measurements were made for the radiation mass attenuation coefficient of the adipose (fat) tissue to examine the effects of the radiation reduction in the thoracic and abdominal anatomic regions. All calculations were performed by using Intel ${ }^{\circledR}$ Core $^{\mathrm{TM}}$ i5 CPU $2.71 \mathrm{GHz}$ computer hardware. MCNPX is a general purpose Monte Carlo code used for modeling radiation interactions between different radiation types and materials. The validation of MCNPX Monte Carlo code for investigation of radiation mass attenuation coefficients for different types of materials such as nanomaterials, glasses, concretes, mixtures, and living biological tissues have been investigated in the literature [17-24]. MCNPX input file has definitions of cell card, surface card, material card, features of energy sources, and positions of each component which form the simulation geometry.

\subsection{Forming geometry for the calculation of radiation mass attenuation coefficients}

To calculate the radiation mass attenuation coefficients, the defined tissues (brain, lung, liver, and adipose tissue) were modeled with MCNPX code and Fig. 1 shows the simulation model for calculating the radiation mass attenuation coefficients of brain, lung, liver, and adipose tissues. The simulation design and screenshot obtained from visualization tool of MCNP can also be seen in Fig. 2.

As shown in Fig. 2, the energy source is located between the lead collimators. The sample material is positioned in the lead collimator and a detector system is placed behind the sample cell. The measurements were performed using mono-energetic beam point isotropic radiation source. The isotropic source is defined in the radiological 0.08, 0.10, 0.12, 0.14, 0.511, 0.662, 1.173, 1.274, and $1.333 \mathrm{MeV}$ energies, respectively. In this study, F4 average flux tally has been employed to quantify absorbed dose in detector area. The elemental mass fractions of each tissue and the densities of the tissues were used in defining the tissue sample cell.

\subsection{Forming geometry for the calculation of radiation attenuation of anatomical $C T$ regions}

Cells and surfaces are defined in the MC input file while the cranium, thorax, and abdomen anatomical region geometry is constructed. Material identifications were

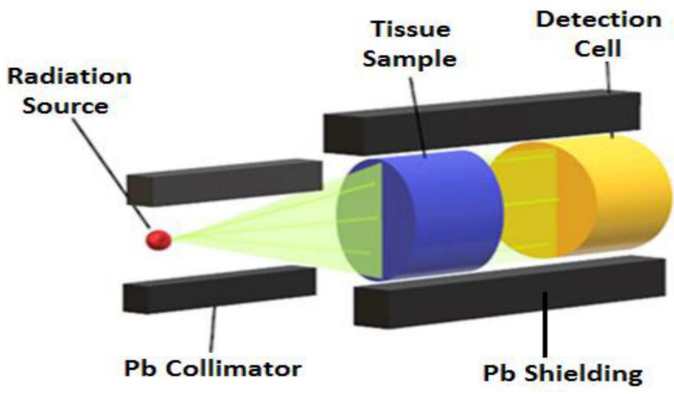

Fig. 1. Total simulation geometry to calculate mass attenuation coefficient of tissue samples.

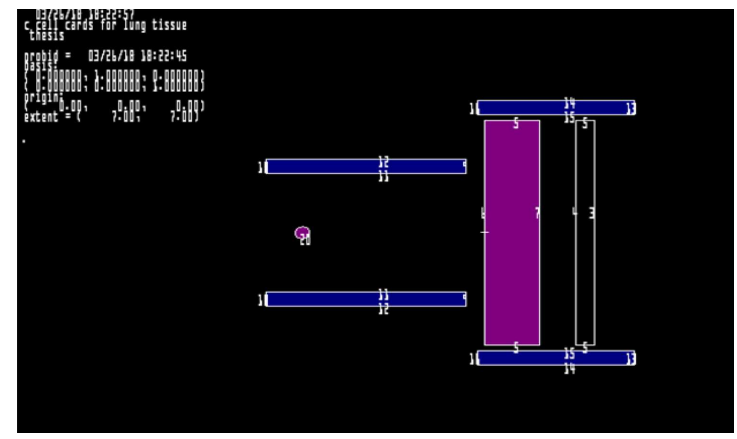

Fig. 2. Screenshot of MCNPX simulation setup. 
TABLE I

Elemental fractions and densities of tissues in cranium, thorax and abdomen anatomical regions [25, 26].

\begin{tabular}{l|c|c|c|c|c|c|c|c|c|c|c|c}
\hline \hline & $\mathrm{H}$ & $\mathrm{C}$ & $\mathrm{N}$ & $\mathrm{O}$ & $\mathrm{Na}$ & $\mathrm{Mg}$ & $\mathrm{P}$ & $\mathrm{S}$ & $\mathrm{Cl}$ & $\mathrm{K}$ & $\mathrm{Ca}$ & $\begin{array}{c}\text { Density } \rho \\
{\left[\mathrm{g} / \mathrm{cm}^{3}\right]}\end{array}$ \\
\hline brain (adult) & 10.7 & 14.5 & 2.2 & 71.2 & 0.2 & - & 0.4 & 0.2 & 0.3 & 0.3 & - & 1.04 \\
cranium & 5.0 & 21.2 & 4.0 & 43.5 & 0.1 & 0.2 & 8.1 & 0.3 & - & - & 17.6 & 1.61 \\
skin (adult) & 10.0 & 20.4 & 4.2 & 64.5 & 0.2 & - & 0.1 & 0.2 & 0.3 & 0.1 & - & 1.09 \\
lung (healthy) & 10.3 & 10.5 & 3.1 & 74.9 & 0.2 & - & 0.2 & 0.3 & 0.3 & 0.2 & - & 0.26 \\
hearth & 10.4 & 13.9 & 2.9 & 71.8 & 0.1 & - & 0.2 & 0.2 & 0.2 & 0.3 & - & 1.05 \\
vertebrae & 7.0 & 28.7 & 3.8 & 43.7 & - & 0.1 & 5.1 & 0.2 & 0.1 & 0.1 & 11.1 & 0.1 \\
ribs & 6.4 & 26.3 & 3.9 & 43.6 & 0.1 & 0.1 & 6.0 & 0.3 & 0.1 & 0.1 & 13.1 & 1.41 \\
breast & 10.6 & 33.2 & 3.0 & 52.7 & 0.1 & - & 0.1 & 0.2 & 0.1 & - & - & 1.02 \\
liver & 10.2 & 13.9 & 3.0 & 71.6 & 0.2 & - & 0.3 & 0.3 & 0.2 & 0.3 & - & 1.06 \\
pancreas & 10.6 & 16.9 & 2.2 & 69.4 & 0.2 & - & 0.2 & 0.1 & 0.2 & 0.2 & - & 1.04 \\
gall bladder & 10.8 & 6.1 & 0.1 & 82.2 & 0.4 & - & - & - & 0.4 & - & - & 1.026 \\
stomach & 10.4 & 13.9 & 2.9 & 72.1 & 0.1 & 0.1 & 0.2 & - & 0.1 & 0.2 & 1.05 & 1.05 \\
kidney & 10.3 & 13.2 & 3.0 & 72.4 & 0.2 & - & 0.2 & 0.2 & 0.2 & 0.2 & 0.1 & 1.05 \\
spleen & 10.3 & 11.3 & 3.2 & 74.1 & 0.1 & - & 0.3 & 0.2 & 0.2 & 0.3 & - & 1.06
\end{tabular}

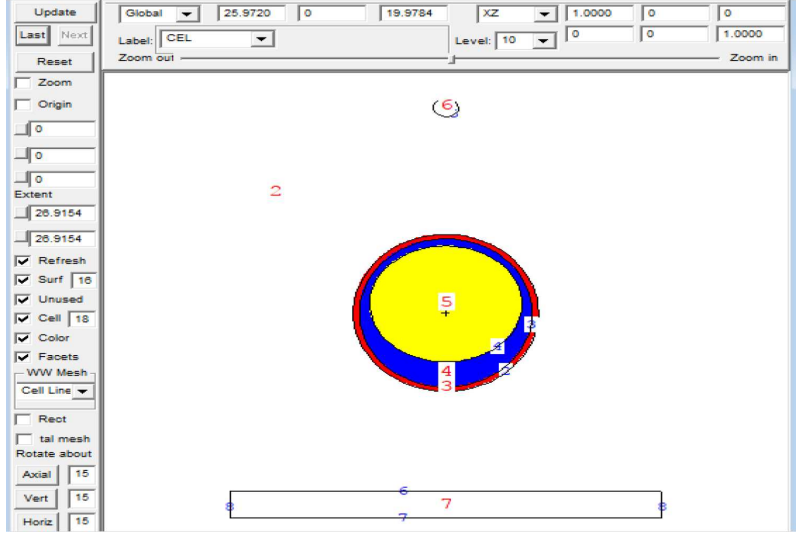

Fig. 3. Cranium anatomic region simulation in $\mathrm{MC}-$ NPX Visual Editor Version X22S.

performed with the tissue/organ elemental fractions and densities. In the present study, some organs and tissues which are placed in the cranium, thorax and abdomen anatomical region, respectively, have been defined considering the elemental mass fractions and densities are given in Table I.

The cranium region simulation design obtained from visualization tool of MCNP can also be seen in Fig. 3. Radiation attenuation quantities of $\mathrm{CT}$ anatomic region of the cranium were determined and measurements were taken by increasing the axial brain diameter from $17.6 \mathrm{~cm}$ to $19.4 \mathrm{~cm}$ by $0.6 \mathrm{~cm}$ steps. The frontal and back view of the body of the thorax and abdominal regions obtained in MCNPX Visual Editor Version X22S is shown in Figs. 4 and 5 , respectively.

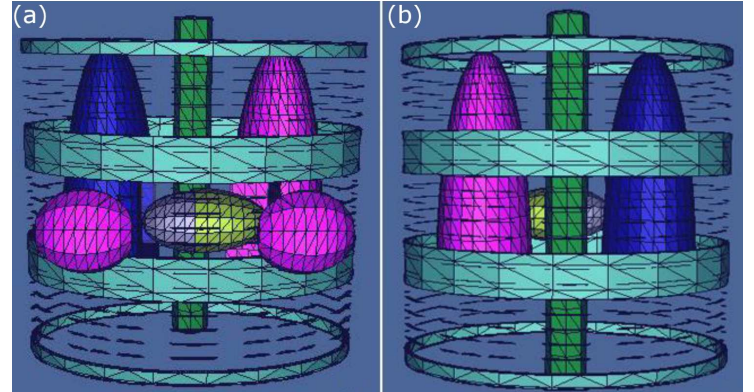

Fig. 4. Thorax anatomic region (except skin layer) from the (a) front, and (b) back of the body in MCNPX Visual Editor Version X22S.

\subsection{Validation of $M C N P-X$}

To calculate the radiation mass attenuation coefficients, the defined tissues were modeled with MCNPX and the radiation mass attenuation coefficients in the radiological energy ranges $(0.08,0.10,0.12,0.14,0.511$, $0.662,1.173,1.274$, and $1.333 \mathrm{MeV})$. The results were compared with standard XCOM data.

\section{Results}

The radiation mass attenuation coefficients of the brain, lung, liver, and adipose tissues at 0.08, 0.10, $0.12,0.14,0.511,0.662,1.173,1.274$, and $1.333 \mathrm{MeV}$ were calculated by MCNPX and XCOM programs and the following graphs were obtained. Figure 6 shows the MCNPX results and compared with standard XCOM data, respectively. This Fig. 6 shows a validation between MCNPX and XCOM results. 

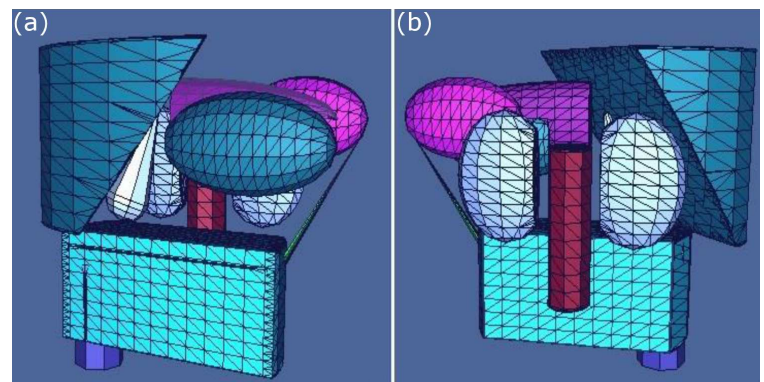

Fig. 5. Abdominal anatomic region (except skin and adipose tissue layer) from (a) front of the body, and (b) back side of the body in MCNPX Visual Editor Version $\mathrm{X} 22 \mathrm{~S}$.
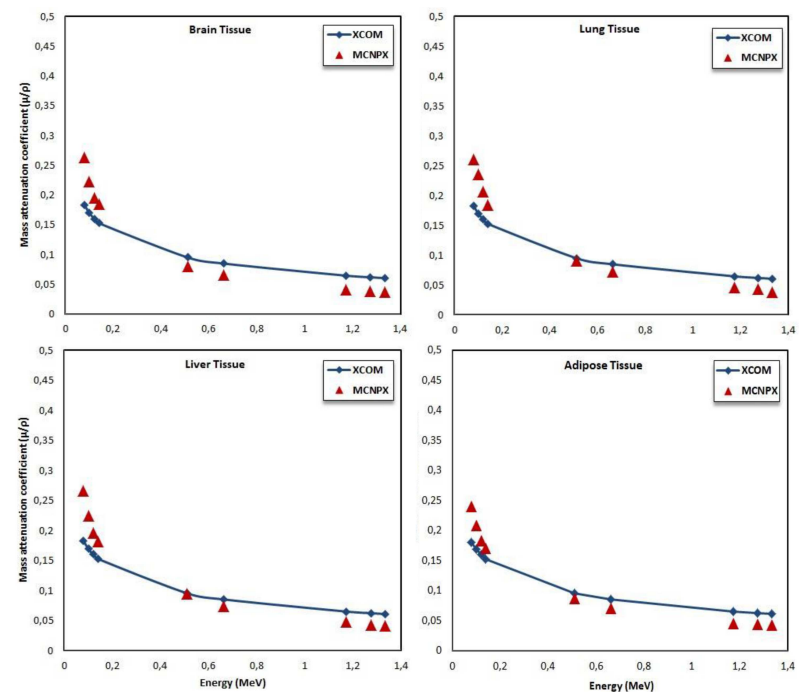

Fig. 6. The mass attenuation coefficients of brain, lung, liver and adipose tissue.

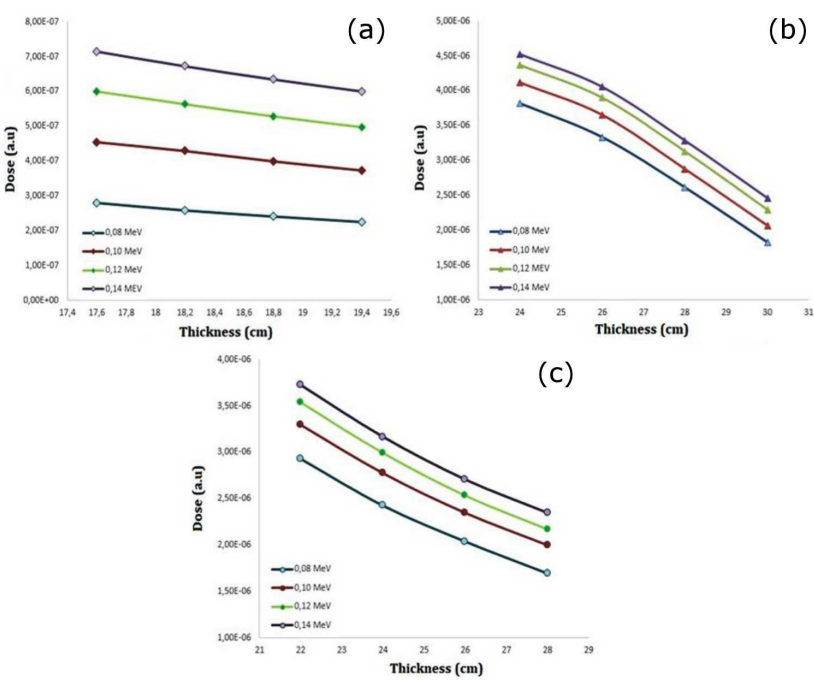

Fig. 7. The amount of dose passing through (a) cranium, (b) thorax, and (c) abdominal anatomic regions at $0.08,0.10,0.12$, and $0.14 \mathrm{MeV}$, depending on the thickness of the regions.
Figure 7 shows the results of changes in radiation attenuation amount depending on the thickness of the brain, thorax, and abdominal regions for CT measurements at $0.08,0.10,0.12$, and $0.14 \mathrm{MeV}$. Slight differences have been obtained between MCNPX and XCOM results. This can be due to statistical differences, data library, and the number of particle tracks in the simulation.

\section{Discussion}

The mass attenuation coefficient results obtained by MCNPX simulation of brain, lung, liver, and adipose tissues and standard XCOM data were compared (Fig. 6). This match is important for the validation of the generated MCNPX input. In Fig. 7a, it is clear that the measured dose decreases due to the thickness of the cranium anatomic region. In Fig. $7 \mathrm{~b}$, it can be seen that the increased volume of breast tissue in thorax region due to the physiological or anatomical structure of the patient, or due to its weight, increases the absorbed dose in the thorax region and causes the dose of radiation reaching the detectors to decrease. This study for the thorax region was associated with adipose tissue thickening in the abdominal region due to obesity. In Fig. 7c, it is can be seen that thickening of adipose tissue in the abdominal region has increased the radiation attenuation coefficient. In Fig. 7, it is seen that there is an inverse proportion between the thickness of the CT anatomic region and the amount of radiation reaching the detector through this region. The amount of radiation reaching the detector is reduced due to the increased thickness through which the beam passes. Comparison of the energies shows that, as the amount of radiological energy used increases, the amount of radiation passing through the region increases. At the same radiation energy value, more energy is delivered to the detectors with low thickness.

\section{Conclusion}

In CT imaging protocols, specific radiation doses are applied to each anatomical region and to the physiological state of the patient. In some special cases, like obesity, the thickening of adipose tissue due to obesity in the abdominal region increases the radiation attenuation coefficient and in this case, the aim is to create CT images with high resolution and high image quality with more higher radiation beam. However, this dose, which is increased due to adipose tissue, also increases the amount of radiation the patient is exposed to and causes more dose absorptions in the anatomical region of the patient. With the ALARA (as low as reasonably achievable) principle, considering the possible biological effects of radiation, these results should be considered in cases where CT dose parameters should be increased. The optimal dose range for optimal quality and patient safety must be selected with optimization. 


\section{Acknowledgments}

This work has been supported by Süleyman Demirel University Foundation Unit (BAP) under project number of $4900 \_$YL2_ 17.

\section{References}

[1] H.O. Tekin, T. Manici, Nucl. Sci. Techn. 28, 95 (2017).

[2] I. Akkurt, S. Kilincarslan, C. Basyigit, Ann. Nucl. Energy 31, 577 (2004).

[3] H. Tekin, M.I. Sayyed, E.E. Altunsoy, T. Manici, Digest J. Nanomater. Biostruct. 12, 861 (2017).

[4] H.O. Tekin, V.P. Sing, T. Manici, E.E. Altunsoy, J. Radiat. Protect. Res. 42, 154 (2017).

[5] J. DeMarco, T. Solberg, J.B. Smathers, Med. Phys. 25, 1 (1998).

[6] H. Yoriyaz, M.G. Stabin, A. dos Santos, J. Nucl. Med. 42, 662 (2001).

[7] B.M. Stoeckelhuber, T. Leibecke, E. Schulz, U.H. Melchert, C.U. Bergmann-Koester, T. Helmberger, J. Gellissen, Cardiovasc. Intervent. Radiol. 28, 589 (2005).

[8] M. Salvado, M. Lopez, J.J. Morant, A. Calzado, Radiat. Protect. Dosim. 114, 364 (2005).

[9] P.H.R. Peixoto, J.W. Vieira, H. Yoriyaz, F.R.A. Lima, Phys. Med. Biol. 53, 5343 (2008).

[10] S. Morato, C. Garcia, B. Juste, R. Miro, G. Verdu, Conf. Proc. IEEE Eng. Med. Biol. Soc. 2017, 560 (2017).

[11] H.O. Tekin, V.P. Singh, E.E. Altunsoy, T. Manici, M.I. Sayyed, Iran. J. Med. Phys. 14, 229 (2017).

[12] O. Günay, Ö. Gündoğdu, M. Demir, M. Abuqbeitah, D. Yaşar, S. Aközcan, E. Kapdan, O. Yarar, IJCESEN 5, 119 (2019).
[13] Y. Yahşi Çelen, H. Orhan Kizilkaya, IJCESEN 5, 142 (2019).

[14] O. Günay, M. Sarihan, E. Abamor, O. Yarar, IJCESEN 5, 86 (2019).

[15] H. Ozan Tekin, B. Cavli, E. Ebru Altunsoy, T. Manici, C. Ozturk, H. Muammer Karakas, IJCESEN 4, 37 (2018).

[16] Y. Yahşi Çelen, H. Orhan Kizilkaya, IJCESEN 5, 135 (2019).

[17] H.O. Tekin, Sci. Technol. Nucl. Installat. 2016 , 6547318 (2016).

[18] I. Akkurt, H.O. Tekin, A. Mesbahi, Acta Phys. Pol. A 128, 332 (2015)

[19] H.O. Tekin, V.P. Singh, U. Kara, T. Manici, E.E. Altunsoy, Celal Bayar Univ. J. Sci. 12, 195 (2016).

[20] H.O. Tekin, T. Manici, V.P. Singh, Politeknik Dergisi 19, 617 (2016)

[21] H.O. Tekin, V. Singh, T. Manici, Appl. Radiat. Isotopes 121, 122 (2017).

[22] M. Dong, R. El-Mallawany, M.I. Sayyed, H.O. Tekin, Radiat. Phys. Chem. 141, 172 (2017).

[23] H.O. Tekin, M.I. Sayyed, T. Manici, E.E. Altunsoy, Mater. Chem. Phys. 211, 9 (2018).

[24] G. Lakshminarayana, S.O. Baki, K.M. Kaky, M.I. Sayyed, H.O. Tekin, A. Lira, I.V. Kityk, M.A. Mahdi, J. Non-Cryst. Solids 471, 222 (2017).

[25] F.A. Duck, Physical Properties of Tissues: A Comprehensive Reference Book, Academic Press, 2013.

[26] G. White, I. Wilson, "Photon, Electron, Proton and Neutron Interaction Data for Body Tissues", ICRU-46 Report, Bethesda (MD) 1992. 